\title{
Capacity of Non Ionic and Ionic Surfactants for Solubilisation of Paracetamol
}

\author{
Tamanna Rahaman, Jannatul Fardous, Faria Farzana Perveen and Sakina Sultana \\ Department of Pharmacy, Jahangirnagar University, Savar, Dhaka-1342, Bangladesh.
}

\begin{abstract}
The present study was conducted to investigate the solubilising capacity of polyvinylpyrrollidone (PVP K-30), Polyethylene glycol (PEG 6000), Polysorbate (Tween 80) and Sodium lauryl sulphate (SLS) for paracetamol. In our study, PVP K-30 exhibited 5 times, Tween 80 showed 3 times and SLS displayed 2 times higher solubilising capacity than water. Here, PVP K-30 exhibited the highest solubilising capacity and the value was $6.36 \pm 0.063$ (mg /ml), whilst PEG 6000 in the same study failed to show any significant increase ( $>0.05$, unpaired t-test, two-tailed).
\end{abstract}

Key words: PVP K-30, PEG 6000, Tween 80, Sodium lauryl sulphate, Solubilising capacity, Paracetamol

\section{Introduction}

Drug solubilising technique designated for enhancing apparent aqueous solubility of hydrophobic drugs by using surfactants has been practiced since many years and it is still being conducted world-wide (Arnarson and Elworthy, 1980; Porter, 1994; Pater, 1998; Sultana, 2000; Ahmed et al., 2003; Sultana et al., 2005). Surfactants are compounds that are important for our lives. For instances surfactants are present in our lungs and makes us alive. The essential building blocks (like Proteins, Lecithin, Cholesterol) of our body have surfactant properties. In modern era, surfactants are being used in targeted drug delivery systems and this has opened a gateway for site specific therapy. Such diversity of uses of surfactant molecules has been successful because of structural uniqueness. Chemically these molecules consist of a polar head moiety and a non polar tail part which consist of a long (straight or branched) hydrocarbon chain. Because of such structural diversity surfactant molecules in solvents forms molecular aggregates known as 'micelles'(Figure 1).

These micelles are formed in a continuous mood beyond critical micelle concentration (conveniently called 'cmc'). Micelles act like micro-containers into which drugs gets entrapped and are carried conveniently to the target sites and elicit drug action. Such physical entrapment of drugs into the micellar system increases apparent solubility of drugs and this is the underlying concept of 'drug solubilisation'. Since earlier days ay investigation regarding surfactant propelled drug solubilisation has been an area of interest. In line of such

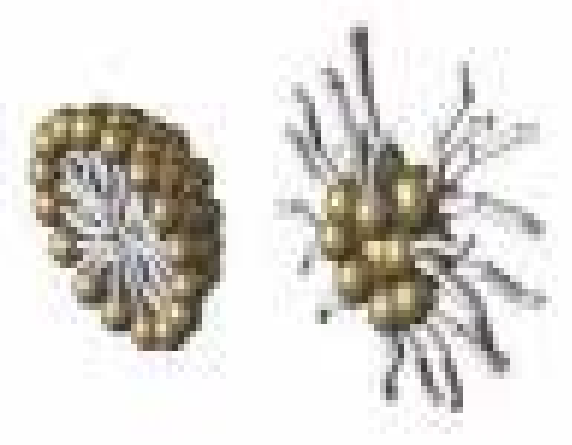

Figure 1. A pictorial presentation of micelles.

study this work was carried out giving preferences on nonionic and ionic surfactants. Characteristically nonionic surfactants as name suggests lacks ionic properties. These molecules do not release ions in to the surrounding fluid and are non toxic, non irritant and bio compatible. Polyvinylpyrrollidone K-30 (PVP K-30), Polyethylene glycol 6000 (PEG 6000), and polysorbate (Tween 80) are the three commonly used non ionic surfactants. Each of these surfactants has got pharmaceutical and medicinal values. For instances, PVP is widely used as formulation stabilizer and drug solubiliser. Secondly, this agent is used as a thickening- and wetting agents. Note that wetting of

Correspondence to: Sakina Sultana, E-mail : sultana_s2003@yahoo.co.uk, 
water insoluble drugs in the GIT is a prerequisite for rapid drug action (A PhA, 1986; Robinson et al., 1990). While PEG is highly recognized for its surfactant property and beneficial medicinal uses, this agent retards mechanical haemolysis, changes the inhibition pattern of response activation and can be used for cancer therapy. In pharmaceutical fields. this it successfully used for wetting and for enhancing viscosity. Thus, uses of PEG 3350 in colonoscopy and in chronic constipation have been well documented (A PhA, 1986; Pashankar et al., 2003a,b; Sultana, 2012). The same is true for Polysorbate 80. It is reported that Polysorbate 80 promotes solubilization of water insoluble drugs besides improving formulation stability (A PhA, 1986; Ismail et al., 1970). Sodium lauryl sulphate (SLS) on the other hand is the most commonly used anionic surfactant and this has been successfully used in formulations of tablets as binder and diluent as well as in the preparation of dentrifices. Earlier studies showed that SLS improved the solubilisation of many chemical agents including argon (Newton and Razzo, 1977; Wilkinson and Moore., 1982; Oates and Schetcher, 1989; Serra et al., 1995). This present study was therefore aimed at investigating solubilising capacity of these surfactants for paracetamol (PCT). In the study PCT was given preferences as it has an established metabolic profile and it has gained the utmost popularity as an analgesic and antipyretic agent (Hardman and Limbird, 1996; Shahidi, 2006). To the best our knowledge studies on solubilising behavior of surfactants for PCT have not been done yet.

\section{Materials and Methods}

\section{Materials}

Polyvinylpyrrollidone K-30 (Merck), Polyethylene glycol 6000 (Merck), and polysorbate (Merck), Sodium lauryl sulphate were obtained from our departmental store, Jahangirnagar university, Paracetamol was a gift from Ganobishawbiddalaya.

\section{Methods}

Drug solubilisation study was conducted at normal day temperature following the method as described by Arnarson and Elworthy (1980), where the model drug (a few mcg) was mixed with the aqueous surfactant solution $(2 \%, 5 \mathrm{~mL})$ for $48 \mathrm{~h}$ using a rotary mixture at a slow rpm.
Assuming the state of equilibrium was attained, the mixture was centrifuged for 2 mins at a speed of 2000 rpm. A definite quantity of the clear, supernatant of the drug-surfactant dispersions was removed and following dilution with methanol, absorbence was determined using a UV/visible spectrophotometer (Shimadzu 160 1PC) at $249 \mathrm{~nm}$. The amount of drug solubilised was then obtained from the standard curve drawn with absorbance versus concentration.

\section{Results and Discussions}

Results are shown in different Figures 2-5. Each figure represented PCT solubilisation in each surfactant and water. In the study, except PEG 6000 a significant increase in PCT solubilisation due to surfactant was evident ( $\mathrm{p}>0.05$, unpaired t-test, two-tailed). PVP K-30 in the study solubilised PCT in an amount of $6.36 \pm 0.063$ ( $\mathrm{mg} / \mathrm{ml})$, whist in the same study water solubilised of 1.26 \pm 0.063 (mg $/ \mathrm{ml}$ ) of PCT (Figure 2).

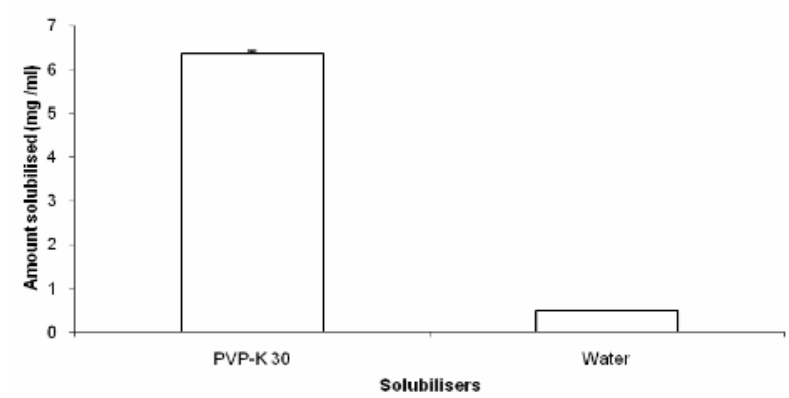

Figure 2. Paracetamol solubilisation in PVP K-30.

Thus, PVP K-30 showed PCT solubilisation almost five times higher than water. Such results were similar to the solubilisation of phenyl butazone, betamethasone, testosterone, cortisone acetate in PVP K-30. And the workers agreed that PVP K-30 increased drug solubilisation in every cases (Sultana, 2000; Sultana and Farooq, 2005). Regarding Tween-80, the present study showed that this agent was to able to solubilise PCT almost 3 folds higher than water as expected (Figure 3).

Ismail et al. (1970) conducted a series of studies with Polysorbates- 80 to study the solubilisation of barbitone, phenobarbitone and cyclobarbitone and they noticed many folds increase in drug solubilisation. Sultana and Farooq (2005) also conducted a similar study on cortisone acetate 
in Tween 80 and observed the same. Regarding SLS study results was not an exception and like the previous studies Tween 80 also increased the solubilisation of PCT (Figure 4).

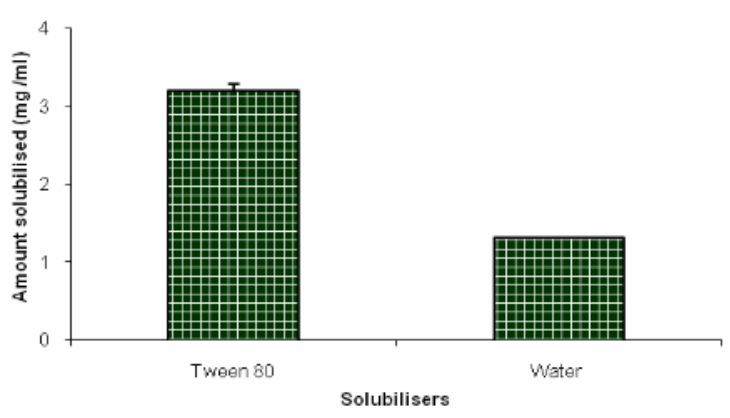

Figure 3. Paracetamol solubilisation in Tween-80.
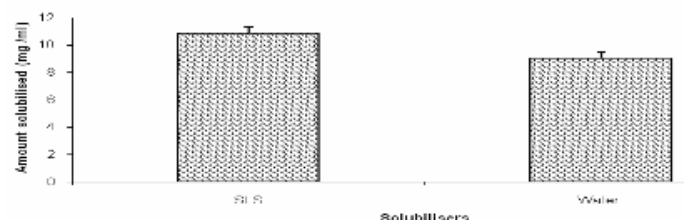

Figure 4. Paracetamol solubilisation in SLS.

Early studies showed that SLS increased solubilsation of argon and other chemicals (Newton and Razzo, 1977; Oates and Schetcher, 1989; Serra et al., 1995). Therefore, results obtained with SLS were as expected. In the study, among the surfactants PVP K-30 showed the highest PCT solubilising capacity followed by Tween 80 and then SLS. Such disparity can be explained by the underlying concept of aggregation theory. It is reported that for a surfactant molecule, the aggregation phenomenon occurs due to the balance between hydrophilic- and the hydrophobic- forces ensuing from the hydrophilic and the hydrophobic moieties, respectively. Usually, in water hydrophobic force encourages aggregation and hydrophilic force disfavours the process (Saito and Sato, 1985; Porter, 1994). Structurally, PVP is a synthetically produced homopolymer of $\mathrm{N}$-vinyl-2-pyrrolidone.and Tween 80 is a polyethoxy sorbitan ester of long chain fatty acid; whereas SLS is the sodium dodecyl sulphate and it bears an alkyl chain of $\mathrm{C}_{12}$ unit. The hydrophobic influence of PVP K-30 and Tween 80 therefore was much higher than SLS and as a consequence promoted micellisation. Therefore, higher PCT solubilisation exhibited by PVP K-30 and Tween 80 as compared to SLS was not unlikely. Interestingly, in the study PEG 6000 failed to show a significant increase in solubilisation of PCT (fig 5).
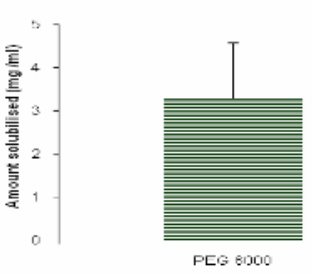

Figure 5. Paracetamol solubilisation in PEG 6000.
Not only that this surfactant exhibited PCT solubilisation less than SLS, this result was similar to Sultana et al. (2003). PEG 6000 is a polymer of ethylene oxide and it may be possible that this surfactant lacks hydrophobicity even less than SLS. Such lacking perhaps resulted inadequate micellisation and hence drug solubilisation (Porter, 1994). However, this needs further studies for confirmation.

\section{Conclusion}

Our study thus concludes that PVP K-30, Tween 80 and SLS were good solubilisers for PCT and among them PVP K 30 was the best.

\section{References}

A. PhA. 1986. Handbook of Pharmaceutical Excipients American Pharmaceutical Association and the Pharmaceutical Society of Great Britain.

Ahmed. J., Rahman. T., and Sultana, S. 2003. Comparison of drug solubilising behaviour between sodium lauryl sulphate and polyethylene glycol. Bangladesh J. Life sci.. 15, 151157.

Arnarson, T and Elworthy, P.H. 1980. Effects of structural variations of nonionic surfactants on micellar properties and solubilisation: surfactants based on erucyl behenyl $\left(\mathrm{C}_{22}\right)$ alcohols. J. Pharm. Pharmacol. 32, 381-385.

Hardman, J.H. and Limbird, L.E (eds). 1996. Goodman and Gilman's The Pharmacological Basis of Therapeutics. $9^{\text {th }}$ edition. MacGraw - Hills, NY.

Ismail, A.A., Wafik, G.M and Motawi, M.M. 1970. Micellar solubilisation of barbiturates I : solubilities of cetain barbiturates in polysorbates of varying hydrophobic chain length. J. Pharmaceut. Sci. 59, 221-223. 
Newton, J.M and Razzo, F.N. 1977. The in-vitro bioavailability of various drugs formulated as hard gelatin capsule. $J$ Pharm. Pharmacol. 29 , 205 - 208

Oates, J.D and Schechter, R.S. 1989. Effect of solubilisation of $\mathrm{CO}_{2}$ on the micellar concentration of sodium alkyl sulfonates. J. Colloid. Interface Sci. 131, 307-310

Pashankar, D.S, Bishop, W.P and Loening-Baucke V. 2003b. Safety of Polyethylene glycol 3350 for the treatment of chronic constipation in children. Arch. Pediatr. Adolese. Med. 157 , 661-664.

Pashankar, D.S., Bishop, W.P and Loening-Baucke, V. 2003a. Long term efficacy of Polyethylene glycol for the treatment of chronic constipation in children with or without encopresis. J. Clin. Pediatr. 42, 815-819.

Patel, R. 1998. Novel Polyethylene pyrrolidone-based surfactants: their synthesis and physico-chemical properties. Ph.D. thesis, University of London, London.

Porter, M.R. 1994. Handbook of Surfactants. Blackie Academic and Professional - Chapman and Hall, London.

Robinson, B.V., F.M. Sullivan and S.L. Schwartz. 1990. PVP-a critical review of the kinetics and toxicology of polyvinylpyrrolidone (Povidone). Lewis Publishers Inc., Michigan.
Saito, Y and Sato, T. 1985. Effects of Polyoxyethylene chain length on micellar structure. J. Phys. Chem. 89, 2110-2113.

Serra, M.C., Coelho, J.A.P; Calado, J.C.G. and Palavra, A. M.E. 1995. Solubility of argon in micellar aqueous solutions of sodium dodecyl sulphate. J. Colloid. Interface Sci. 173, 278-283

Shahidi, R.U. (ed). 2006. Quick Index of medical Products and Problems (QUIMP). Dhaka, Bangladesh.

Sultana, S. 2000. Comparison between polyvinyl pyrrolidonebase and polyoxazoline nonionic surfactants : their physicochemical and solubilisation behaviour. Ph. D thesis. Department of Pharmacy, King's College London, University of London.

Sultana, S. 2012. Evaluation of wetting property and purgation inducing capacity of nonionic surfactants in mice. Jahangirnagar Univ. J. Biol. Sci. 1, 77-80

Sultana, S.; Farooq Omar A.T.M., Kamruzaman, Md. and Imtiaz, M.F. 2011. Solubilising capacity of PEG 6000, PVP 30 and Polysorbate 80 for Cortisone acetate. J. Bangladesh Soc. Pharmace. Profess. 1, 51-54.

Wilkinson, J.B and Moore, R.J (eds). 1982. Hrry’s Cosmetology. $7^{\text {th }}$ Edition. Longman Scintific and Technical Publishers, England. 